\title{
Humanización del cuidado de Enfermería
}

MCE. Gloria Inés Prieto Parra*

*Profesora Titular de la Facultad de Enfermería Universidad de la Javeriana. Bogatá, Colombia.

Como marco de referencia para el análisis del tema se hará inicialmente una reflexión acerca del cuidado de Enfermería el cual se fundamenta en la relación interpersonal humanizada que establece la Enfermera con el sujeto de cuidado; así mismo se analiza el enfoque humanístico del cuidado teniendo como referente lo planteado por algunas teoristas en Enfermería. Finalmente se proponen posibles caminos que contribuyan a la humanización del cuidado para lo cual se han tenido en cuenta los valores y los principios éticos que guían el desempeño de la Enfermera en Colombia, los derechos del sujeto de cuidado y la importancia del trabajo en equipo de las personas que participan en la atención de salud de quienes la requieren.

PALABRAS CLAVES: Cuidado de Enfermería, humanización, persona, dignidad humana.

\section{Abstract}

In order to analyze the issue, the initial step is a reflexion of the nursing care, which is based on the interpersonal relation between the nurse and the care subject, besides the human approach of the care is analyzed, regarding to some of the nursing theorizers. Finally is proposed some possible paths to humanize the patient care, for which is taking into account the ethic values and principles that guides the nurse practice in Colombia, the patient rights and the importance of team work of the persons that take part in health attention for the one who need it.

KEY WORDS: Nursing care, humanization, person, human dignity.

\section{INTRODUCCIÓN}

Los avances de la ciencia y la tecnología aplicadas al campo de la salud han llevado a un mayor conocimiento de la naturaleza, causas y tratamientos de las patologías que el hombre padece; así mismo han surgido nuevos marcos de referencia acerca de la vida, la muerte, la reproducción asistida, la herencia genética y la eugenesia.

Estos cambios han ocasionado una mayor complejización de la atención en salud. Hasta hace pocas décadas ésta era prestada principalmente por el médico y la Enfermera, la relación que se establecía con el sujeto de atención era más directa, individualizada, de persona a persona. En la actualidad se observa que para atender a una persona enferma se requiere de diversos profesionales, cada uno especializado en una parte del organismo, se da más importancia a los costos que genera una hospitalización y a descubrir el significado de los exámenes diagnósticos que a la persona a quien se le realizan, deshumanizándose así su atención.

Dichos cambios también han traído como consecuencia que el profesional enfrente continuamente situaciones complejas que le generan dilemas éticos, es decir que sus principios y valores entran en conflicto y él tiene que decidir sobre que es lo correcto, lo más beneficioso y justo para la persona o grupos a quienes brinda atención en salud. 
Ante esta situación el tema de la humanización de los servicios de salud tiende a ser cada vez más importante; los trabajadores de la salud en general y las diferentes profesiones, entre éstas Enfermería, lo incluyen como un aspecto fundamental en la búsqueda del mejoramiento de la calidad del cuidado de la salud que se brinda a la sociedad. Aquí es conveniente clarificar que el tema de la humanización en salud es un referente básico para toda persona que se desempeña en esta área: profesionales, técnicos, auxiliares y demás trabajadores por tanto no es un referente exclusivo de los profesionales del área de la salud.

\section{CUIDADO DE ENFERMERÍA Y HUMANIZACIÓN}

Todas las personas cuidan y esta actividad humana, como dice Domínguez Alcón, ha sido entre otras una expresión de cariño, de amor a otros, significa interés, preocupación, afecto, ayuda, compasión.; en sentido genérico y aplicado a Enfermería se refiere a aquellos actos de asistencia, de soporte, de ayuda, de conservación que facilitan a la persona o grupos con necesidades, el poder mejorar sus condiciones de vida o anticiparse a esas necesidades; todo ello en una relación de participación. ${ }^{1}$

Se entiende por CUIDADO todas las acciones que realiza el profesional de Enfermería con el fin de incentivar o potencializar los recursos individuales y colectivos de la persona o del (los) grupo (s) a que pertenece, de manera que se constituya en un agente central, activo, decidido y autónomo de su propia salud. Torres considera que el cuidado de Enfermería, como actividad profesional, está mediado por rasgos humanos y por componentes científicos y tecno- instrumentales, implica una acción que tiene sentido y está orientada hacia la búsqueda del bienestar de la persona o grupo que se cuida. Afirma que el desafío actual de la Enfermería es desarrollar y mantener la dimensión humana del cuidado pues nos encontramos "en una sociedad cuyos valores están centrados en la tecnología, el dinero, la estrategia del mercado y las organizaciones administrativas eficientes donde la dignidad humana parece no tener la misma dignidad"2

Ahora bien teniendo como referente los avances que en la conceptualización del cuidado han logrado diferentes grupos de estudio conformado por Enfermeras de servicio y docentes, el acto de cuidado de Enfermería se ha definido como "el ser y la esencia de la profesión. Se fundamenta en sus propias teorías y tecnologías y en conocimientos actualizados de las ciencias biológicas, sociales y humanísticas. Se da a partir de la comunicación y relación interpersonal humanizada entre el profesional de Enfermería y el ser humano, sujeto de cuidado, la familia o grupo social, en las distintas etapas de la vida, situación de salud y del entorno.

Implica un juicio de valor y un proceso dinámico y participativo para identificar y dar prioridad a las necesidades y decidir el plan de cuidado de Enfermería, con el propósito de promover la vida, prevenir la enfermedad, intervenir en el tratamiento, en la rehabilitación y dar cuidado paliativo con el fin de desarrollar, en lo posible, las potencialidades individuales y colectivas."3

Dicha relación interpersonal humanizada, se constituye en la base del cuidado humanizado y este en la concepción que se tenga de persona, dado que la humanización hace referencia al proceso de comunicación y apoyo mutuo entre las personas, orientado hacia la autotransformación y hacia el entendimiento mutuo, es aplicable a cualquier aspecto del desempeño humano entre estos al cuidado de Enfermería.

Para profundizar en el concepto de persona es necesario establecer las diferencias entre individuo y persona, ya que la diferenciación entre estos dos conceptos, que no son contrapuestos sino que se constituyen en dos niveles en el ser humano, puede hacer más fácil la comprensión del segundo de ellos. En sentido general la individualidad es lo que hace que una cosa de la misma naturaleza que otra, difiera de esta otra en el seno de la misma especie. El hombre y los animales son individuos, esto es poseen la propiedad de la individualidad que separa unos de otros dentro de su misma especie y género. El individuo de la especie humana es una unidad vital en un momento dado de su desenvolvimiento temporal, es uno distinto en un grupo o en una fila.

Cuando se habla de persona, en sentido general, se hace referencia al ser humano en sus relaciones con el mundo y consigo mismo. La filosofía reconoce que todo hombre es persona, es un ser integral, totalitario, único e irrepetible que involucrado en un contexto particular piensa, siente y actúa de acuerdo a un conjunto propio de valores, creencias, prácticas, características y experiencias vividas a lo largo de la vida que lo hace mucho más de lo que a simple vista es observable. La persona tiene características que la distinguen de otros seres vivos de la naturaleza.

\section{CARACTERÍStICAS de LA PERSONA}

- La persona es un sujeto espiritual, solo las personas son capaces de hacer juicios de valor, de ahí que 
Scheler haya expresado: "Todo acto que realiza un valor debe ser definido precisamente como manifestación de una persona."

- La persona permanece durante el curso de nuestra vida, independiente de los períodos por los que se pase, en este sentido se dice que es intemporal. Un ser humano en el transcurso de su vida puede tener muchos cambios (físicos, espirituales, sociales, psicológicos), sin embargo sigue siendo él mismo, la misma persona.

- La persona tiene el derecho, supremo e inalienable, de regir su vida, de formarse un mundo interior propio y de elegir lo que crea conveniente entre las diferentes opciones que se le presentan. Como ser racional tiene la capacidad para orientar sus propios actos y de sentir y actuar por sí mismo en forma libre y autónoma.

- La persona es interioridad que alcanza a trascender es decir a salir de sí hacia el exterior, a través del conocimiento y del amor. Está abierta a todo y esta apertura hace que perciba en forma diferente a los demás y al mundo que la rodea. ${ }^{4}$

- La persona tiene dignidad, esto es una condición particular, un rango que la coloca por encima del resto de los seres vivientes, es superior a todo precio. Por poseer dignidad es merecedora de respeto y de que se le proteja el derecho a vivir como ser humano.

Cely analiza el concepto de dignidad en dos dimensiones: la ontológica y la existencial ${ }^{5}$. La primera viene siendo la marca de fábrica de la especie humana: ontológicamente somos dignos; por el solo hecho de ser seres humanos, de pertenecer a le especie humana; de allí dimanan todos los derechos humanos. La dimensión existencial hace referencia a la manera como se cualifica la dignidad en cada persona, creciendo o disminuyendo en atributos. Esto significa que cada ser humano hace sus propios méritos (virtudes) que aportan tonalidad a su dignidad ontológica; o lo contrario puede deteriorar su dignidad ontológica si su conducta personal es contraria al comportamiento esperado por la cultura. Afirma el autor:

"Vivir éticamente es un agregado necesario de la dignidad existencial a la dignidad ontológica"

Con respecto al concepto de dignidad, Erizari considera que el conocimiento de sí mismo es el fundamento de la dignidad de la persona lo cual implica: Valoración de sí mismo, compromiso de querer realizarse y creatividad para el logro de sus metas. ${ }^{6}$

Haciendo una aplicación de los conceptos anteriores al campo de la Enfermería, se encuentra que a través de la historia de la misma el centro del cuidado de Enfermería ha sido la persona. Es así como algunas teoristas han profundizado en la dimensión humanística del cuidado de Enfermería. Dentro de ellas se encuentran: Florence Nightingale, Faye Glenn Abdellah, Ernestine Wiedenbach, Virginia Henderson, Lydia Hall, Myra Levine, Jean Watson, Patricia Benner, William Hobble, Theresa Lasinger y Callista Roy. ${ }^{7}$

A manera de síntesis se citará el enfoque fundamental de cada una de dichas teoristas por considerar que aportan elementos para la Humanización del Cuidado de Enfermería.

Florence Nightingale sustentaba que toda mujer velaba por la salud de alguien en algún momento, siendo por lo tanto una cuidadora de la vida. Consideraba que la enfermedad era un proceso reparativo y que la Enfermera debía manejar el medio ambiente del paciente para facilitar el proceso, manteniendo una adecuada ventilación, calor, luz, dieta, limpieza y disminución del ruido. Nightingale defendía que las Enfermeras eran agentes morales y presento una guía para la relación profesional con los pacientes; inculcó a sus Enfermeras el principio de confidencialidad y les recomendó cuidar a los pobres para mejorar su salud y su condición social.

Faye Glenn Abdellah, en la década del cincuenta, basada en el análisis de la situación de Enfermería planteaba que la formación y el ejercicio profesional se enfrentaba a grandes problemas como consecuencia del desarrollo tecnológico y del cambio social y que el desempeño de la Enfermera orientado por las funciones médicas resultaba inadecuado para el desarrollo de la profesión. Ante esta situación y a finales de la década de los sesenta (1969) Abdellah plantea la Tipología de los 21 problemas de Enfermería los cuales sirvieron de base para construir el cuerpo de conocimientos de su teoría de Enfermería, el método de solución de problemas es la base de su modelo.

En sus escritos más recientes (finales de la década del ochenta) Abdellah define Enfermería como un servicio a los individuos, familias y sociedad, es hacer algo para la persona y su familia, o suministrar información a éstas para identificar sus necesidades, elevar o restablecer la capacidad de autoayuda o aliviar el sufrimiento. Apoya el método holistico de asistencia centrada en el paciente y 
la necesidad de conceder más importancia a los factores del entorno y a la promoción de la salud.

Ernestine Wiedenbach centra su teoría en las interacciones entre la Enfermera y el paciente a quien define como "cualquier individuo que recibe ayuda de cualquier tipo, bien sean cuidados, instrucciones o avisos procedentes de un profesional de la salud, en este caso la Enfermera, por lo tanto para ser un paciente no se necesita estar enfermo". Considera que tanto la Enfermera como el paciente son seres humanos, dotados de potenciales únicos, que luchan por la autodeterminación y que como tal actúan, piensan y sienten. Plantea además, que la Enfermera cuya actividad está dirigida a prestar cuidado de acuerdo a la necesidad de ayuda identificada en el paciente, debe aprender a disciplinar sus pensamientos y sentimientos.

Virginia Henderson basada en sus estudios, experiencias e investigaciones define la Enfermería en términos funcionales, para ella la función de la Enfermera es ayudar al individuo enfermo o sano, en la realización de aquellas actividades que contribuyan a su salud, o a su recuperación (o a una muerte tranquila), y que él realizaría sin ayuda si tuviera la fuerza, voluntad o conocimientos necesarios.

Lydia Hall, su planteamiento acerca de Enfermería se centra en tres componentes: Cuidado, introspección y curación. Para ella cuidado significa alimentar y se refiere a la asistencia corporal íntima del paciente y lleva implícita una relación reconfortante y educativa. Introspección se refiere a la utilización terapéutica del YO en la comunicación con el paciente; en este sentido la Enfermera debe pensar antes de preguntar y ayudar al paciente a aclarar sus necesidades y objetivos, facilitando el proceso de autoconocimiento. Curación es el componente de la Enfermería relacionado con la administración de medicamentos y tratamientos prescriptos por el médico o por otros profesionales. Hall considera que los tres componentes están interrelacionados, y un componente precede los otros dos en diferentes momentos del progreso del paciente.

Myra Levine ve la Enfermería como una interacción humana donde existe dependencia de los individuos unos a otros. Enfatiza que las interacciones y las intervenciones de Enfermería tienen como fin "conservar la unidad y los recursos individuales que cada persona que se encuentra en dificultades aporta a la situación". Utiliza cuatro principios de conservación para describir las acciones de Enfermería: Conservación de la energía, conservación de la integridad estructural, conservación de la integridad personal relacionada con la autoestima y el sentido de la identidad y conser- vación de la integridad social. En este sentido, para Levine la vida adquiere significado a través de los grupos sociales y por tanto la salud está determinada socialmente.

Jean Watson en su libro: Human Science and Human Care. A Theory of Nursing (1985, reedición 1988) considera que el cuidado es la esencia del ejercicio profesional de la Enfermería y que el objetivo del mismo es "facilitar que las personas adquieran un grado superior de armonía entre la mente, el cuerpo y el alma; que de lugar a los procesos de autoconocimiento, autorreverencia, autocuración y autoasistencia". Afirma que este objetivo se logra mediante un proceso de relación interhumana y mediante actividades de asistencia que faciliten el desarrollo de los pacientes en el ámbito de la promoción de la salud mediante acciones preventivas.

En la reedición de su libro (1988) analiza los aspectos relacionados a los valores del cuidado humano en Enfermería y afirma "El cuidado humano puede ser demostrado efectivamente y ser practicado solo de manera interpersonal. El proceso humano intersubjetivo mantiene vivo un sentido común de humanidad, él nos enseña como ser humanos al identificarnos nosotros mismos con los demás de manera tal que la humanidad de cada uno se refleje en el otro"8

Patricia Benner describe Enfermería como una relación de asistencia en la cual se crea la posibilidad de dar y recibir ayuda, su ciencia está guiada por el arte moral, la ética de la asistencia y por la responsabilidad. Entiende el ejercicio profesional de la Enfermería como la asistencia a la persona y el estudio de sus experiencias vividas de salud, dolencia y enfermedad y las relaciones que existen entre ellas. Benner retoma la descripción fenomenológica de Heidegger sobre la persona "Una persona es un ser que se interpreta así mismo, es decir la persona no se incorpora al mundo predefinida, sino que se define a lo largo de la vida..."

Callista Roy. Moreno hace un análisis del Modelo de Adaptación de Roy y destaca que los supuestos filosóficos del mismo se asocian con los principios del humanismo y de la veritivity. ${ }^{9}$ El humanismo considera esencial conocer y valorar todas las dimensiones de la persona. Roy considera que las personas como individuo o en grupos comparten un poder creativo, su existencia tiene un propósito, poseen un holismo intrínseco, buscan mantener la integridad y se dan cuenta de la necesidad de mantener relaciones con los demás. Veritivity es un término acuñado por Roy y hace referencia al propósito común de la persona humana que transciende hacia lo espiritual, a la actividad y creatividad que busca el bien de todos y al valor y significado de la vida. 
Como se deduce de las Teorías de Enfermería descritas anteriormente el fin de Enfermería es brindar Cuidado a la persona, familia y grupos de la comunidad basado en una relación interpersonal que permita la humanización del mismo. Al respecto William Hobble y Theresa Lansinger basadas en el trabajo de Joyce Travelbee plantean un MODELO DE RELACION INTERHUMANA que representa la interacción que se establece entre la Enfermera y la persona o familia que cuida. Dicha relación se establece mediante un proceso que se desarrolla en diferentes etapas:

- Encuentro original. Se caracteriza por las primeras impresiones obtenidas por la Enfermera sobre la persona enferma y de la persona enferma sobre la Enfermera. Los dos se perciben mutuamente dentro de los papeles esteriotipados que se le asignan a cada uno.

- Revelación de identidades. Se caracteriza por la percepción que tiene uno del otro como individuos únicos, a partir de ella surgen los primeros lazos de la relación.

- Empatía. Capacidad de compartir las experiencias de la otra persona y de predecir su conducta. Se facilita cuando hay experiencias similares y existe el deseo de entender a la otra persona.

- Simpatía. Se produce cuando la Enfermera desea aliviar la causa de la enfermedad del paciente o su sufrimiento. "Cuando alguien simpatiza está implicado, pero no se ve incapacitado por esta implicación".

- Acercamiento. Ocurre cuando la Enfermera y la persona enferma se relacionan como un ser humano con otro ser humano. "La Enfermera es capaz de conseguir el acercamiento porque posee los conocimientos necesarios y las actitudes necesarias para ayudar a las personas que requieren de su cuidado y porque es capaz de percibir, apreciar y responder a la individualidad de cada una de ellas como seres humanos".

¿Cómo se aplican estos elementos de la relación interpersonal en el quehacer cotidiano de la Enfermera?

En la formación profesional se hace énfasis en que la Enfermera debe considerar a la persona o grupos que cuida como seres humanos, sin embargo y como lo menciona Garzón "Infortunadamente, en ocasiones el contexto de la práctica y el sistema de organización no permiten

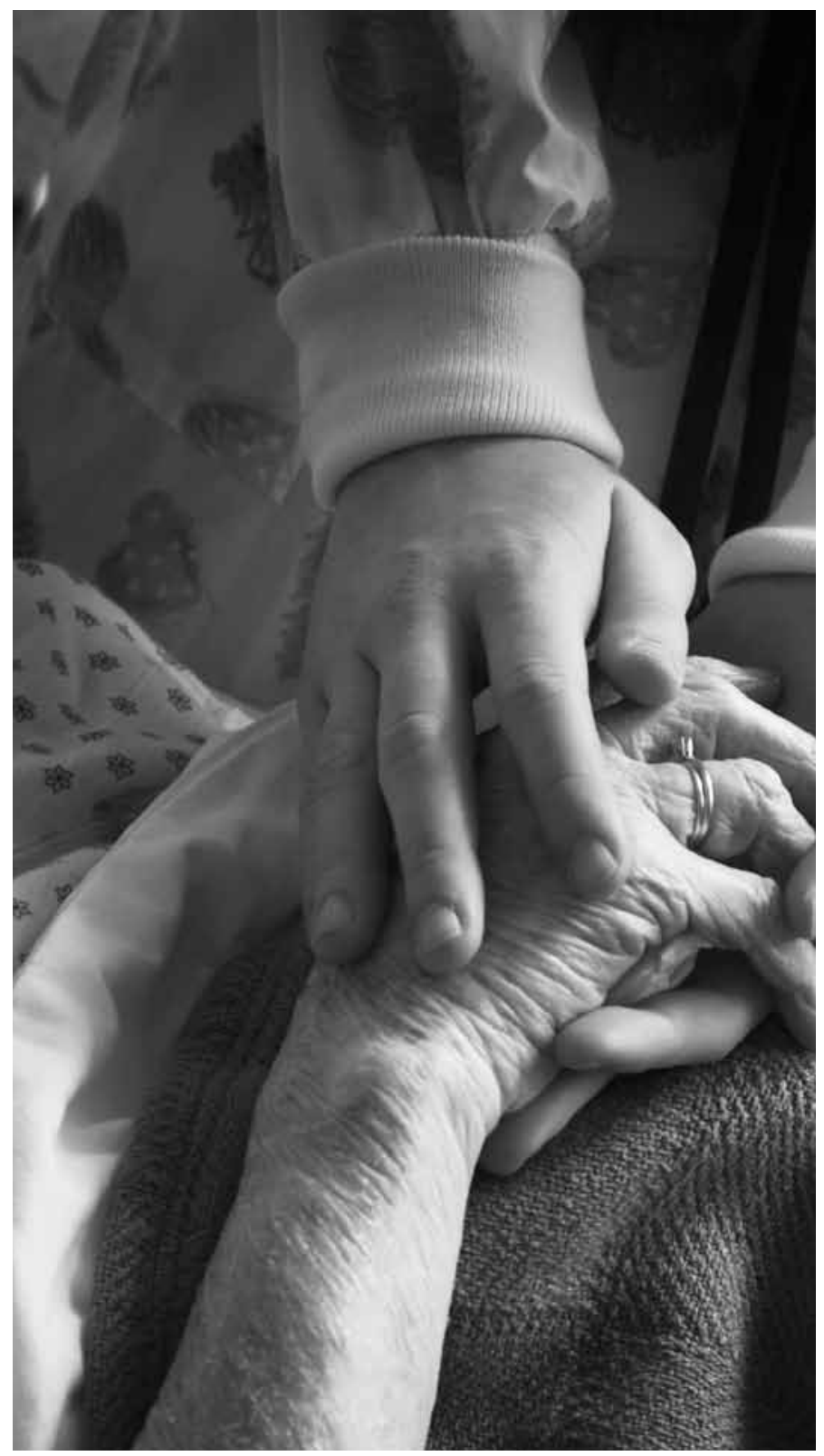

mantener la coherencia esperada entre el saber ético y el saber de Enfermería que debe traducirse en las prácticas de cuidado, y por tanto, la percepción de la persona que recibe el cuidado lo califica como mal cuidado o cuidado des-humanizado, al sentir o percibir que no se tienen en cuenta su valor y sus derechos como persona." 10

En este sentido es usual observar que en la práctica diaria como el ser humano que requiere de nuestro CUIDADO pasa a ser "la historia 475", "el número 503", "la cama 8", "la toxémica", "la diabetes descompensada" "el monito de la cama 10" etc. en vez de ser "el señor Daza" o "la señora Marta" que presenta determinada situación de salud o que requiere determinados cuidados. Al nominar a la persona por un número la individualizamos pero a la vez la despersonalizamos y al despersonalizar, des- 
humanizamos. De ahí la necesidad imperiosa de profundizar en el ser y esencia de nuestra profesión: El cuidado a la persona.

\section{CAMINOS DE HUMANIZACIÓN DEL CUIDADO DE ENFERMERÍA}

Teniendo en cuenta los planteamientos hechos hasta aquí, la vivencia profesional y algunas observaciones de la práctica de Enfermería acerca de la relación que el profesional estable con el sujeto de cuidado, me permito proponer algunos caminos o estrategias que buscan contribuir a la humanización del cuidado de Enfermería, son éstos:

- $\quad$ Fortalecer el conocimiento de si mismo. El reflexionar constantemente sobre nuestros actos nos permite identificar las fortalezas y debilidades que como seres humanos tenemos. Esto permite mantener comportamientos favorables y establecer estrategias para cambiar los que deben mejorar. Así mismo orienta la coherencia entre lo que se piensa, siente y hace. Se considera que el conocimiento de sí mismo fortalece la relación humana entre las personas.

- Reflexionar acerca de los valores y principios éticos que guían la práctica de Enfermería para que se constituyan en el eje central del quehacer cotidiano del profesional de Enfermería. En este sentido es importante conocer, profundizar e introyectar el marco ético legal que orienta la formación y ejercicio de la Enfermera.

- Mayor interés por los derechos humanos, los derechos del paciente y de su familia. Como lo plantea Sara Fry la Enfermera en muchas ocasiones se constituye en la abogada del paciente y/o de su familia, en este sentido es la defensora y protectora de su dignidad humana, intimidad, decisiones y de sus derechos y esto no puede limitarse a una simple repetición y proclamación de los mismos. ${ }^{11} \mathrm{Se}$ requiere de un análisis reflexivo de cada uno de ellos, ya sea de forma individual o colectiva, de tal manera que el cuidado que se brinda no se centre solamente en la ley como tal sino en los principios éticos y valores en que se fundamentan dichos derechos.

- No categorizar a la persona sana o enferma, la familia o grupos que se cuidan de acuerdo a su nivel social, al estrato o entidad de salud a que pertenecen, todos son seres humanos y como tal tienen dignidad y valor que lo hacen merecedor de estima- ción, respeto y de la misma calidad de cuidado. El hacerlo es una forma de discriminación es decir de exclusión social que amenaza y vulnera el derecho a la igualdad y a la equidad; lo cual afecta la dignidad humana de la persona o de los grupos.

- Incentivar la formación permanente; una institución es dinámica y humanizante en la medida en que se promueven oportunidades de actualización para el personal. Sin formación no hay renovación y crece el descontento, la desmotivación y la rutina. Lopera considera que los actos de los profesionales de la salud son morales cuando se: clarifican los valores y principios personales, conocen y reflexionan los valores morales profesionales consignados en los códigos deontológicos y los valores morales sociales consignados en las leyes y en las tradiciones y además cuando el profesional busca mantenerse actualizado en el conocimiento científico y en la tecnología para que los actos que realiza contribuyan al bienestar de la persona (principio de beneficencia) y no le hagan daño (principio de no maleficencia) ${ }^{12}$

- Aplicación de la ética comunicativa. En la relación Enfermera - sujeto de cuidado es fundamental la aplicación de la ética comunicativa o dialógica de Habermas en la cual la comunicación debe ser libre de coacciones y toda persona se respeta como interlocutor válido que tiene derecho y capacidad para razonar y participar en las decisiones por ejemplo en las relacionadas con el cuidado de su salud.

- Tomar decisiones éticas. La relación entre ética y humanización reviste una importancia fundamental en el mundo de Enfermería. El profesional como se mencionó anteriormente, continuamente se enfrenta a dilemas éticos los cuales requieren ser analizados teniendo como referencia los principios, valores y normas éticas. Al tomar decisiones la Enfermera siempre se debe preguntar iestán a favor de la persona, de su desarrollo, de sus derechos, de su calidad de vida?

Fortalecer el trabajo en equipo. La diversidad de criterios y puntos de vista sobre la situación de la persona, familia o grupo que requieren cuidado en salud implica un trabajo coordinado entre las personas que lo prestan, éste nace del reconocimiento de los propios límites, frente a la complejidad del sufrimiento humano, y de la necesidad de valorar la aportación de diversas intervenciones. 
- Profundizar en la dimensión humana del cuidado de Enfermería más que en las técnicas que se utilizan para proveerlo. Algunos profesionales encuentran mayor satisfacción en la realización de técnicas complejas y el grado de reconocimiento que ello proporciona ante otros profesionales que en la contribución que dicha técnica tenga en el CUIDADO a la persona.

\section{CONCLUSIONES}

Los avances en las ciencias y tecnologías aplicadas a la salud han contribuido a un mayor conocimiento de las enfermedades que afectan a la persona sin embargo en su aplicación se prioriza, en la mayoría de casos, el beneficio económico para las instituciones y profesionales implicados y no la calidad de atención integral que se debe proporcionar a la persona que la requiere.

Desde los orígenes de la Enfermería el cuidado ha sido considerado como el ser y la esencia de la profesión, a través de la evolución de la misma se ha observado el interés por profundizar en las dimensiones del cuidado siendo una de ellas la humanística que se basa y hace evidente en la relación interpersonal que establece la Enfermera con el sujeto de cuidado.

Tanto la Enfermera como el sujeto de cuidado son personas con características, derechos y valores que los hacen únicos e irrepetibles y en la relación entre ellos se establece un vínculo humano que facilita el intercambio mutuo de vivencias, experiencias, y conocimientos que orientan el cuidado de Enfermería acorde a las necesidades del sujeto de cuidado.

El fortalecimiento del cuidado de Enfermería humanizado requiere de cambios a nivel personal, profesional e institucional y de un trabajo reflexivo individual y grupal que permita diseñar y aplicar prácticas de cuidado que hagan evidente la dimensión ética y humanística del cuidado.

\section{REFERENCIAS BIBLIOGRAFICAS}

1. Domínguez Alcón, Carmen. "La percepción del cuidar", en Revista Rol de Enfermería. No 127. Barcelona, 1997, p. 27

2. Torres, Ana María y otros. Experiencia de Conceptualización en Enfermería. Facultad de Enfermería. Pontificia Universidad Javeriana. Centro Editorial Javeriano, CEJA, Bogotá, 2001, p. $32-33$

3. Tribunal Nacional Ético de Enfermería. Ley 911 de 2004. Por la cual se dictan disposiciones en materia de responsabilidad deontológica para el ejercicio de la profesión de Enfermería en Colombia; se establece el régimen disciplinario correspondiente y se dictan otras disposiciones, Bogotá, 2004. p. 15

4. Castillo, Valery y otros. La Ética y la Moral en la práctica de Enfermería. Electiva I. Escuela de Enfermería. Facultad de Medicina. Universidad Central de Venezuela. Caracas, 1995.

5. Cely Galindo, Gilberto S. J. Ethos vital y dignidad humana. Colección Bioética. Editorial JAVEGRAF; Bogotá, 2004, p. 190-191.

6. Elizari Basterra, Francisco Javier. Bioética. Madrid: Ed. Paulinas; Madrid, 1991, p. 56.

7. Marriner Tomey, Ann y Raile Alligood, Martha. Modelos y Teorías de Enfermería. 5a edición. Ed. Elsevier. Madrid, 2003.

8. Watson, Jean. Enfermería: Ciencia Humana y Cuidado Humano: Una teoría de Enfermería. Adaptación y traducción Beatriz Sánchez. Nacional League for Nursing, USA, 1988. p. 31 - 36.

9. Moreno Fergusson, María Elisa. Importancia de los modelos conceptuales y teorías de Enfermería: Experiencia de la Facultad de Enfermería de la Universidad de la Sabana en Revista Aquichan. Año 5. Vol. 5 № 1. Facultad de Enfermería Universidad de la Sabana. =Chía. Colombia, octubre 2005, p. 50

10. Garzón Alarcón, Nelly. Ética profesional y teorías de Enfermería. En la Revista Aquichan. Año 5. Vol. 5. № 1. Facultad de Enfermería. Universidad de la Sabana. Chía, Colombia, octubre 2005. p. 71.

11. Fry, Sara. La Ética en la práctica de Enfermería. Ginebra: Consejo Internacional de Enfermería. Ginebra, 1994. p. 49 - 51

12. Lopera de Peña, Angela. Ética y Bioética. Orion Editores Ltda. Bogotá, 2005. p. 45.

\section{DIRECCIÓN PARA CORRESPONDENCIA:}

Mtra. Gloria Inés Parra: gloriaromano@etb.net.co

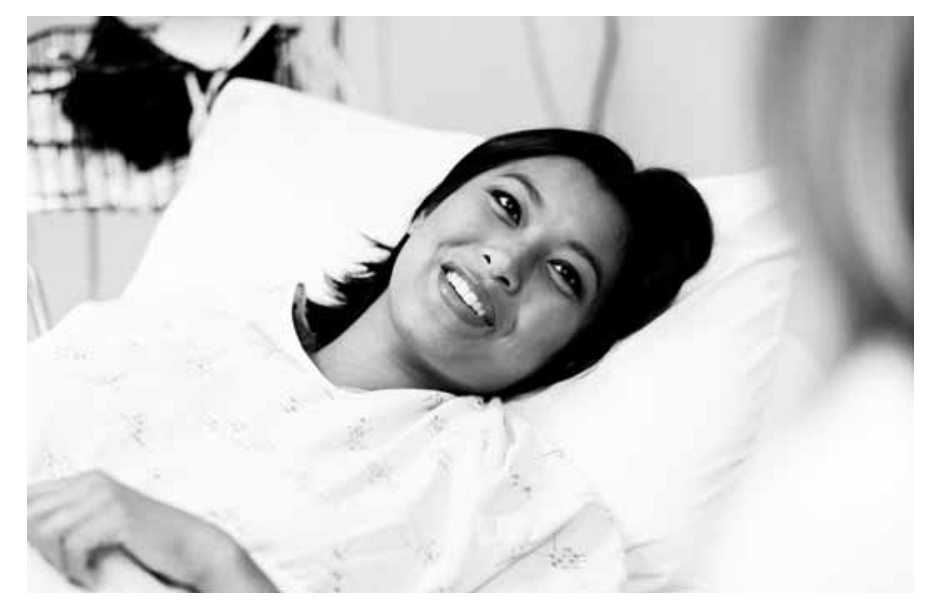

